\title{
MARAÑAS CUERPOS/SILLAS/NEOLIBERALISMO EN LA EDUCACIÓN INICIAL DE CHILE POST-PINOCHET
}

Entanglements chairs/bodies/neoliberalism in post-pinochet chilean early childhood education

Emaranhados corpo/cadeiras/neoliberalismo educação na inicial pós- pinochet chile

\section{Sarita Galvez Donoso}

\author{
Monash University, Australia. Fono: +61 047918 4527. Correo electrónico: \\ sarita.Galvez@monash.edu
}

\begin{abstract}
Resumen
En el siguiente artículo propongo compartir una serie de ideas que he trabajado los últimos años en relación a cuerpo y educación, si bien estas observaciones pueden ser extendidas a la educación en general, mi foco es la educación inicial. Mi enfoque teórico está enmarcado en el feminismo materialista, o los nuevos materialismos donde destacan pensadoras como Karen Barad, Rosi Braidotti, Donna Haraway, Hillevi Lenz Taguchi, entre otras. La propuesta es hilar el concepto de intra-acción de Karen Barad con el concepto de Enacción del biólogo y pensador chileno Francisco Varela. Con este hilo analizaré la maraña sillas $\Leftrightarrow$ cuerpos $\Leftrightarrow$ neoliberalismo $\Leftrightarrow$ Chile utilizando como caso de estudio la campaña 'En el jardín aprendí' de la Fundación Integra (2014). Para finalizar propondré nudos de pensamiento donde las hebras antes señaladas, se mezclan con el contexto geopolítico de Chile post-Pinochet desde dónde emergen.

Palabras clave: sillas; neoliberalismo; cuerpos; maraña; posthumanismo.
\end{abstract}

\begin{abstract}
In the following article I propose a series of ideas that I have been developing in the past years in relationship with bodies and education. Even though, many of these observations could be applied in general education, mi focus is early childhood education. I drawn to Feminist Materalisms or New Materialisms as my main theoretical framework with thinkers as Karen Barad, Rosi Braidotti, Donna Haraway,
\end{abstract}


Hillevi Lenz Taguchi, among others. My proposal is to spin Barad's concept of intraaction with Enactive cognition a concept proposed by the Chilean thinker and biologist Francisco Varela. With this thread I will analyse entanglements in Fundación Integra's media campaign 'what I learnt in preschool' (2014). To conclude I will propose knots of thought where these threads will get entangled with the geopolitical context of postPinochet Chile.

Key words: chairs; neoliberalism; bodies; entanglements; posthuman.

\section{Resumo}

No artigo a seguir proponho para compartilhar algumas idéias que têm trabalhado nos últimos anos em relação ao corpo e à educação, embora estas observações pode ser estendido para a educação em geral, meu foco é a educação da primeira infância. Minha abordagem teórica é enquadrado no feminismo materialista, ou novos materialismos que incluem pensadores como Karen Barad, Rosi Braidotti , Donna Haraway, Hillevi Lenz Taguchi, entre outros. A proposta é girada o conceito de intra -action Karen Barad com o conceito de enação biólogo chileno e filósofo Francisco Varela. Com esta lista de discussão Vou analisar o sillas $\Leftrightarrow$ cuerpos $\Leftrightarrow$ neoliberalismo $\Leftrightarrow$ Chile emaranhado como um estudo de caso utilizando a campanha "No jardim eu aprendido" (2014) Integra Foundation. Finalmente vou propor nós de pensamento, onde os fios acima mencionados são misturados com o contexto geopolítico de pós- Pinochet Chile, de onde emergem.

Palavras chave: cadeiras; o neoliberalismo; corpos; emaranhado; pós-humanismo.

\section{Introducción}

En los últimos 20 años la educación inicial ha cobrado una relevancia sin precedentes a nivel mundial (Dalhberg and Moss, 2005, Malone, 2007, Duhn, 2014, Canella, 1997). Dahlberg y Moss (2005) destacan la masiva institucionalización de la niñez temprana, a través del aumento de la cobertura de salas cunas y jardines infantiles producto del incremento en la inversión en la niñez (notable en los países de la OECD). Nunca antes en la historia de la humanidad más niños y niñas de los países del Occidente habían pasado más tiempo en instituciones (Ibid). ¿De dónde viene este interés desmesurado 
por la niñez?, ¿quién se beneficia? ¿a quién se protege? ¿cómo? ¿cuál es el ideario político tras estas propuestas? Si bien, no ahondaré en la compleja trama que sostiene las posibles respuestas, es necesario mencionar que ellas se basan en el ideario humanista liberal (algunos dirían antropocéntrico, ) que prima en los países de la OECD, y una lógica de resultados planificados/esperables que produce la certeza que si los países invierten A y hacen B, obtendrán C. Siendo C un individuo autónomo, competente con amplias posibilidades de empleo en el sistema que lo produce. El niño y la niña se convierten, por lo tanto, en una inversión en una lógica causa-efecto, donde la educación se propone moldearlos y prepararlos como seres humanos para el cambio (Dalhberg and Moss, 2005).

De manera interesante, Dahlberg y Moss evitan posicionarse desde la nostalgia por tiempos cuando los niños y niñas pasaban más tiempo con sus familias en la casa o el barrio. No obstante, son enfáticos en la necesidad de analizar críticamente los discursos dominantes, para así abrir espacios para que otras posibilidades puedan emerger desde la co-existencia de múltiples discursos. Chile neoliberal es un interesante ejemplo para analizar estos fenómenos discursivos. En su análisis Galdames (2011), señala que es necesario cuestionar el supuesto éxito y equidad de la educación inicial en Chile en los últimos 20 años (Umayahara, 2006). Galdames propone decolonizar el campo de la educación parvularia, a través del análisis y re-conceptualización de ciertas paradojas, que ponen de manifiesto la presencia de trayectorias Neo-coloniales, que operan como discursos incuestionables embebidos en la lógica del ideario neoliberal. El análisis de Galdames, recuerda la frase del filósofo francés Guilles Deleuze 'Si las protestas de los niños y niñas fueran oídas en el Jardín infantil, si sus inquietudes fueran atendidas, sería suficiente para hacer explotar el sistema educacional completo' (Deleuze, 1980 citado en Olsson 2009).

Galdames agrega complejidad al escenario, haciendo notar que estos múltiples discursos Neo-coloniales y neoliberales se interceptan en la realidad local con discursos de raza, clase y principalmente el discurso de infancia. Éste discurso originado en Occidente y ampliamente criticado desde el campo de estudios de la niñez y educación parvularia (Canella, 1997, Kehaly, 2013, Dalhberg y Moss, 2005) concibe la niñez como período separado y distinto de la adultez, dónde el niño y niña se encuentra en un estado de inocencia, apolítico, asexual, que debe ser resguardado y protegido para evitar 
la contaminación del mundo adulto, y además está permeado por la psicología del desarrollo que provee 'la evidencia científica' que establece estadios de desarrollo inicial que obedecen a una lógica de progreso hacia la adultez como el estado avanzado de pensamiento racional, autonomía y orden. Canella (1997) es certera en su análisis, al destacar el aporte que este discurso ha tenido en la vida de muchos niños y niñas, no obstante, es clara al asumir que a través del mismo se reproducen dinámicas de control y extrema vigilancia de la niñez.

En resonancia con Tesar (2016) percibo la urgencia de movernos del análisis discursivo, genealógico e histórico como único espacio reflexivo, hacia la necesidad de redescubrir en la materia un espacio válido y fértil para re-conceptualizar prácticas educativas. En sociedades como Chile, dónde las políticas de libre mercado impulsadas durante la dictadura militar (Letelier, 1977) y consolidadas en la democracia neoliberal (Undurraga, 2015, Muñoz, 2007) han marcado el tejido social y la producción de una sociedad de consumo, resulta crucial definir el materialismo desde la mirada feminista o los nuevos materialismos.

\section{Feminismos materialistas}

Los feminismos materialistas, son una corriente de pensamiento que emergió en el siglo XXI en diversas áreas incluyendo la filosofía, teoría cultural, feminismo, estudios de la ciencia, artes, entre otros. Y se define alrededor de la primacía de la materia, sus propiedades y acciones, explorando los límites entre lo animado, no animado, las agencias, etc. La teórica Karen Barad (2003, 2007, 2015) provee un fundamento teórico clave desde el feminismo al analizar 'difractivamente' ${ }^{1}$ teoría crítica (Foucault), teoría queer (Butler) y física cuántica. Barad argumenta la necesidad de moverse del giro lingüístico de las ciencias sociales y humanidades, puesto que pareciera que lo único que ya no importa (matter en inglés) es la materia (matter). Con este juego de palabras, Barad propone que la materia no es un ente pasivo que está a la espera de la acción humana que actúa 'sobre' ella, si no que la materia también está haciendo. O quizás, más claro sería decir que la materia y el ser están siendo hechos o co-emergen en intra-

\footnotetext{
${ }^{1}$ Barad inspirada en Donna Haraway, utiliza la metáfora de la difracción como fenómeno físico como alternativa al uso de la reflexión. En su opinion, la reflexión corresponde a un efecto de espejo, por otra parte, la difracción permite la generación de diferencia a partir del análisis.
} 
acción. Lo que Barad propone es, por tanto, un enfoque post-antropocéntrico, que nos permite movilizarnos del limitante representacionalismo. Esto se explica a continuación.

Desde la mirada representacionalista quien conoce, es independiente de lo que se conoce y sólo tiene un acceso parcial a la realidad externa a través de representaciones (conocimiento), que crea a partir de una lógica input/output. Barad propone el uso del neologismo intra-acción como alternativa a la interacción, puesto que en la interacción el sujeto y el objeto existen como entidades fijas con límites y márgenes pre-existentes definidos. La intra-acción sugiere que los límites emergen en la relación y no son fijos, si no que se van haciendo y re-haciendo en la historia de lo que Francisco Varela llama la historia del acoplamiento entre el cuerpo y su ambiente. En consecuencia, la propuesta de Barad es relacional, y nos invita a pensar que los cuerpos, los discursos, las sillas, la economía, la política, la geología, las emociones, las bacterias son entanglements o marañas temporo-espaciales, donde la unidad ontólogica es el fenómeno (entendiendo la ontología como el estudio filosófico que se pregunta por la naturaleza de la realidad, en el caso de Barad su propuesta de los postulados de físico Niels Bohr).

Esta onto-epistemología sugiere en tanto, un giro radical en cómo entendemos la realidad y el conocer. Es una propuesta al realismo (existe un mundo allá afuera independiente de nosotros) y al constructivismo (la realidad es construida por quien conoce de acuerdo a su contexto socio-cultural). Es así como un objeto ordinario, como una silla, no es entendido como objeto monolítico, fijo, sino que en las diversas posibilidades de ser/actuar/hacer dependiendo de la maraña dónde emerge y la multiplicidad de intra-acciones simultáneas que ahí suceden. A través del encuentro con múltiples marañas, se abren los espacios para nuevas formas de pensar por qué el Cartesianismo sigue tan enraizado en la educación chilena.

El educador Carlos Calvo $(2004,2011)$ ha teorizado sobre el cuerpo en educación, dando cuenta de la negación constante que sufren los cuerpos en el proceso de escolarización y cómo el cuerpo y movimiento son negados, constreñidos y limitados en su accionar. Calvo es enfático en mencionar que si bien los discursos pedagógicos, a través de planes y programas, parecen progresistas, en la práctica se sigue negando la posibilidad de que conocemos con nuestro cuerpo (Calvo, 2015). En relación al 
conocer, el trabajo de Francisco Varela $(1991,2000,2006)$ fue y sigue siendo relevante, puesto que contribuyó sustancialmente en el campo de la cognición corporizada o radical embodiment, en inglés.

\section{La enacción}

Varela propone que aquello que llamamos 'mente' no está en el cerebro, si no distribuida en el cuerpo, que el cuerpo/mente está embebido en un ambiente, y más aún propone que la 'mente' no está restringida a un cuerpo 'individual', sino que es intersubjetiva. Al plantear que el cuerpo/mente está inmerso en un ambiente, es enfático en hacer notar que este ambiente no es pre-dado si no que emerge a partir del acoplamiento sensoriomotriz del ser que conoce, cuya historia de acoplamiento es relevante para nuestras capacidades de enactuar realidades/mundos (pareciera ser, entonces, que el paso concreto de mundos posibles a mundos probables dependería de esta historia). En otras palabras, el mundo es inseparable de nuestro hacer y simultáneamente, nuestro hacer es inseparable del mundo (con las implicancias éticas de hacerse cargo de ese mundo). Se asevera así, que el individuo y el mundo se crean mutuamente, por lo tanto, no es el objeto separado del sujeto, ni el sujeto que construye el objeto, sino una co-construcción sujeto-objeto, está es la 'vía del medio' propuesta por Varela (2000).

Así como intra-acción es un neologismo acuñado por Barad, enacción también es un neologismo acuñado, en este caso, por Varela. Varela et al. (1991) crea esta palabra a partir del verbo inglés to enact que define como hacer emerger. Similar al concepto de cut-together-apart (cortar-juntos-separados) propuesta por Barad para describir lo que sucede cuando los límites emergen en la intra-acción.Varela sugiere que la cognición 'es traer ese mundo al frente,' y ese proceso es acción corporizada. De esta manera, Varela (2006) propone que a través del acoplamiento sensorio-motor hacemos emerger nuestra realidad, la traemos 'hacia el frente.' Uno de los conceptos cruciales para entender la enacción es la idea de la percepción como acción perceptualmente guíada., es decir.... En mi opinión, entender la percepción como acción nos lleva a entender por qué el movimiento es tan relevante a la hora del conocer.

Hasta acá se han desarrollado ideas que permiten visualizar cómo podría tejerse el pensamiento de Varela con la educación inicial, puesto que gracias al legado de Jean 
Piaget para la mayoría ésta idea resulta familiar, aprendemos en movimiento. La diferencia radical entre ambos, es que para Piaget si bien el período sensoriomotor es fundacional y la base de los estadios siguientes, en una lógica de progreso/desarrollo lineal, en la medida que el niño progresa a la adultez, se mueve desde lo concreto a lo abstracto. Si bien Varela reconoce en el trabajo de Piaget una 'clave esencial' no comparte este pensamiento de progreso lineal de lo concreto a lo abstracto; en otra dirección es enfático en sostener:

"Las verdaderas unidades de conocimiento son de naturaleza eminentemente concreta, incorporadas, encarnadas, vividas; el conocimiento se refiere a una situacionalidad y que lo que caracteriza al conocimiento su historicidad y su contexto no es un "ruido" que oscurece la pureza de un esquema que ha de ser captado en su verdadera esencia, una configuración abstracta. Lo concreto no es un paso hacia otra cosa. Es cómo llegamos y dónde permanecemos” (Varela, 1999: 5)

Es precisamente su foco en la naturaleza concreta del aprender, no como un paso a otra cosa sino como medio y fin, donde quisiera centrar mi análisis. Puesto que este planteamiento nos permite también cuestionar cómo creemos que los llamados 'adultos' aprendemos, y cómo la historicidad y contextos en los cuales hemos aprendido están también inscritos en nuestros cuerpos, y en los mundos que 'traemos al frente' las epistemologías, metodologías y practicas pedagógicas que utilizamos. Hilando entonces, las proposiciones hechas por los materialismos feministas y específicamente, el concepto de intra-acción con la materia y la enacción de Varela, es que las invito a conectarnos con la silla en el contexto de la educación inicial.

\section{Caso de estudio: Campaña \#eneljardínaprendí de Fundación Integra}

La silla ha sido utilizada como símbolo de la educación en Chile en varias ocasiones, algunos ejemplos son el 'Monumento al futuro de nuestros niños,' campaña para dar visibilidad al ausentismo escolar de Fundación Oportunidad (2015), y el Memorial 'Un lugar para la Memoria de Nattino, Parada y Guerrero' conocido como 'Las Sillas' (2006). Así también, es probable que quien entre en intra-acción con estas ideas comience a acceder a la experiencia corporal de su propio cuerpo tras extensos períodos 'aprendiendo ensillados.' La silla está de alguna u otra forma inscrita en nuestra historia de acoplamientos sensoriomotores, un patrón de movimiento limitado y repetitivo pararse, sentarse, pararse, sentarse, en un loop prolongado por años de escolarización. 
En educación inicial, la Fundación Integra entre los años 2010-2014 potenció la silla de diversas maneras, la más frecuente fue a través del uso de una silla de madera en miniatura usada como símbolo de su quehacer educativo. Esta silla se utilizó como reconocimiento a educadoras a través del premio 'Espíritu Integra', en la campaña 'La silla que se transformó en biblioteca' (2010-2011) y la campaña que me dispongo a analizar hoy 'En el jardín aprendí' (2014). Esta campaña mediática se realizó para promover el ingreso de niños y niñas de estratos populares a la educación inicial. De esta manera, esta institución atiende más de 74.000 niños y niñas en todo el territorio nacional, provenientes en un $93,9 \%$ del $60 \%$ de los hogares con menos ingresos en nuestro país (Integra, 2016).

¿Por qué la silla se utiliza como símbolo de educación inicial en una institución que atiende a niños y niñas que están aprendiendo a mover sus cuerpos y ser movidos por el mundo que habitan?, ¿cómo el uso de la silla en el espacio público naturaliza la intra-acción sillas/cuerpos en las salas cunas y jardines infantiles?, ¿por qué se mantiene la relación entre cuerpos quietos y aprendizaje? ¿cómo se intersectan estas preguntas con los discursos de raza y clase analizados previamente?

Resulta relevante hacer una breve referencia a los antecedentes históricos de la Fundación Integra, así como también su quehacer educativo actual. Fundación Integra es una fundación privada sin fines de lucro dependiente del Estado de Chile fundada el año 1990, durante los primeros años de transición a la democracia. Sus inicios se remontan al año 1975, durante la dictadura militar de Pinochet, siendo fundada por Lucía Hiriart como Fundación Nacional de Ayuda a la Comunidad (FUNACO). El objetivo principal durante sus primeros años fue asistencial, entrega de alimentación y ropas a niñas, niños y familias de escasos recursos, así como una función 'normalizadora,' puesto que las voluntarias (en su mayoría señoras de miembros de las fuerzas armadas, o civiles simpatizantes del régimen) también dedicaban tiempo a instruir hábitos de higiene y normas de comportamiento en los menores que atendían (ref).

El año 2006 es un año clave para las políticas públicas de niñez en el Chile neoliberal post-Pinochet, puesto que se aprueba el programa de atención integrado de la infancia "Chile Crece Contigo (CCC)" y junto a él, una serie de medidas que buscan 
"proteger el desarrollo infantil y promover equidad en las condiciones de aprendizaje" (Medrano, 2009: 2). Para lograr este objetivo, la entonces presidenta Michelle Bachelet promociona el slogan "Equidad desde la cuna" poniendo énfasis en la inversión que Chile realiza para proteger y potenciar la niñez. Entre el 2005 y 2007 las salas cuna en Chile aumentaron en un $240 \%$, enfocados principalmente en la atención de los menores de sectores más vulnerables (ibid). Fundación Integra es junto a la Junta Nacional de Jardines Infantiles 'actor principal' para este proyecto expansivo.

La campaña \#eneljardínaprendí podría entenderse a la luz de este esfuerzo estatal por aumentar la cobertura e incrementar el número de menores de 4 años en salas cunas y jardines infantiles. La campaña comprende un video de 3:30 min publicado en canal de Youtube de Fundación Integra, Integravisual en Febrero del 2014. En este video aparecen celebridades de la televisión chilena que comparten lo que aprendieron cuando asistieron al jardín, a partir de la pregunta "¿qué aprendiste en el jardín que te sirvió para toda la vida?”. En el video no hay niños, si no adultos exitosos (en la lógica neoliberal) que juegan con sillas en miniatura, las toman, las acarician, pretenden que los dedos de sus manos son cuerpos de niños, las ubican en relación a partes de su cuerpo, y sonríen. Analizaremos entonces esta campaña usando la hebra que hilamos anteriormente con los conceptos de intra-acción de Barad y enacción de Varela.

\section{Maraña sillas/cuerpos/fundación integra/neoliberalismo/Chile}

Las marañas (mi traducción de entanglements) nos ofrecen una forma de entender fenómenos que incorporan dentro del análisis fuerzas no-humanas y más-que-humanas en intra-acción con lo humano. Se de-centra así el humano de su centro autónomo para entender que somos parte de y siempre en devenir con otros y otras, así las intraacciones nos iluminan ese 'camino del medio' de Varela, para hablar de las coconstituciones, que son múltiples y complejas. Barad es clara en argumentar que las marañas no son 'sólo una forma de interconectar, entrelazar o un enredo en una situación complicada.' (160) La propuesta de la maraña es precisamente demostrar que las entidades no preceden la relación, y que es la relación la que produce conocimiento (Barad, 2003). Al proponer la maraña, Barad plantea una alternativa a la idea Cartesiana de los límites pre-establecidos entre objeto y sujeto. En el caso de la silla y el cuerpo 
humano desde una visión objetiva Cartesiana ambos interactúan, pero no se determinan mutuamente.

Nos dice Barad que la materia no es fija, que su hacer y sus límites dependen de las intra-acciones a través de las cuales los límites emergen y profundiza en cómo los discursos y teorías (desde una perspectiva Foucaultiana) también son materiales. ¿Qué maraña emerge en el video \#eneljardinaprendí? aprendizaje $\Leftrightarrow$ sillas $\Leftrightarrow$ adultos $\Leftrightarrow$ éxito $\Leftrightarrow$ raza $\Leftrightarrow$ clase $\Leftrightarrow$ ropas $\Leftrightarrow$ sonrisas $\Leftrightarrow$ progreso $\Leftrightarrow$ tacto $\Leftrightarrow$ miradas $\Leftrightarrow$ carreras $\Leftrightarrow$ fama $\Leftrightarrow$ joyas $\Leftrightarrow$ maquillaje $\Leftrightarrow$ Fundación Integra $\Leftrightarrow$ infancia $\Leftrightarrow$ expansión $\Leftrightarrow$ neoliberalismo $\Leftrightarrow$ educación $\Leftrightarrow$ Chile $\Leftrightarrow$ neo-colonialismo (utilizo estas flechas siguiendo a Jackson y Mazzei (2012) quienes las han usado anteriormente para mostrar las intra-acciones, y co-constitución de la maraña).

A su vez, siguiendo a Donna Haraway (1988) y la idea de los conocimientos situados, es fundamental hacerse cargo y situarse en la maraña también, puesto que el saber es enmarañado. Como investigadora soy parte también de la maraña que emerge, la afecto y soy afectada simultáneamente por ella. Y así también será para la lectora (o lector), quien traerá la maraña al frente desde su propia posición. En mi caso, no es casualidad que hoy me interese por el fenómeno de las sillas y los cuerpos, desde pequeña me interesé por el movimiento y posteriormente estudié Kinesiología. Nací, aprendí a mover mi cuerpo, aprendí a hablar, caminar y comer en el Chile gris de la segunda década de dictadura militar de Pinochet. El año que nací en Chile había vuelto a regir el estado de sitio. Soy madre de dos niños menores de 7 años, y descubrí con ellos la motricidad global en libertad siguiendo las ideas de Emmi Pikler. Además, crecí en Chile, territorio sísmico, siendo movida constantemente por la tierra. El entender las agencias más-que-humanas y las ideas de mover y ser movida, fue para mí algo natural, siempre en devenir con, siempre parte de. Y como gran parte de los chilenos y chilenas el primer día del jardín me senté y no me paré hasta que egresé de la Universidad. Más de 19800 horas sentada. Mis caderas tienen esa historia de acoplamiento registrada en las fascias anudadas. Para mí estas ideas son profundamente políticas y, siguiendo la ruta del feminismo académico, las pienso a través del cuerpo.

En sintonía con lo planteado por Calvo (2004, 2015), en relación a la negación del cuerpo en la educación, llama la atención cómo en el siglo XXI en Chile se siga 
relacionando un artefacto estático como la silla con el aprender, ¿Qué permite que aún domine la idea de que "se piensa con el cuerpo quieto"? Es poco probable encontrar respuestas únicas a este fenómeno, puesto que como nos asevera Calvo (2015) las respuestas dicotómicas son las más engañosas. En consecuencia, creo que la maraña es productiva para comprender que esta relación no es dicotómica, si no múltiple o polivalente. No es únicamente una mirada opuesta a otra, sino una intersección de simultáneas capas de intra-acciones espacio/temporales dónde el fenómeno se materializa y a su vez, materializa (a otros, otro).

Los discursos de infancia, como bien lo expresa la filósofa Olga Grau (2011) dan cuenta en cómo la infancia ha sido conceptualizada como 'pasividad, pero también un tiempo a la espera de..., un porvenir más que un presente, pura potencialidad.' Resulta interesante, entonces, que las niñas y niños están ausentes en la campaña, como si sus cuerpos en miniatura pudieran ser proyectados a esas sillas de promesas, de éxito, de fortuna. En la lógica de resultados esperables que menciona Dalhberg y Moss (2005) en relación al proyecto neoliberal, el adulto exitoso es el producto del proceso educativo donde el niño se ha modelado, como una posibilidad, una potencialidad. No obstante, esto contrasta con la realidad de un país como Chile, organizado conscientemente por estructura de clases (OECD, 2004).

Relacionando el neoliberalismo y cognición, Blum y Fenton (2016) entregan luces de cómo el dualismo mente/cuerpo ha sido reforzado por el campo de las neurociencias sosteniendo que 'el neoliberalismo y su fascinación con las neurociencias como una autoridad para formar a los niños para la era de las informaciones, en un contexto donde mejores oportunidades para la participación económica asumen un actor hipercognitivo, y cerebro-céntrico' (p. 100, mi traducción). Es precisamente éste reforzamiento de la separación entre mente y cuerpo, a través del discurso científico 'cerebro-céntrico' que vuelven a tomar fuerza prácticas de dualismo encarnado. Volviendo a Dalhberg y Moss (2005) resulta crucial mantener actitud crítica en relación a cómo estos discursos materializan y se enmarañan con otros.

Este análisis de la campaña de Fundación Integra haciendo visible una de las marañas material-discursivas que producen el fenómeno 'aprendiendo ensillados,' invita a atender las intra-acciones entre discursos de clase $\Leftrightarrow$ neoliberalismo $\Leftrightarrow$ silla. Surgen así 
las preguntas ¿a quienes precisamente se busca sentar?, ¿a quien se le ofrece la silla? puesto que como argumenta Galdames (2011) 'Bajo una lógica post-colonial, los niños pobres aún necesitan ser domesticados' (111). El discurso de la niñez vulnerable y en riesgo, constituye un aparato de control de los estratos populares, al generar la idea de la necesidad de intervención para 'potenciar el desarrollo y contribuir a la equidad'. No obstante, cuándo se ofrece una silla, ¿a qué se invita? En mi opinión, esa 'domesticación' a la que se refiere Galdames, guarda estrecha relación con la historia reciente de Chile y las múltiples capas de su suceder. Hay un paralelismo interesante en lo descrito por Calvo (2015) y sus observaciones de las educadoras 'frenando el movimiento' y la estasis crítica en la que se sumió Chile post-Pinochet, dónde la movilización social también es frenada, limitada. Se yuxtaponen así los cuerpos individuales, con los cuerpos colectivos, sociales.

\section{Nudo de pensamiento, 0 (in)conclusiones}

Como mencioné en un inicio, este artículo es inconcluso, principalmente porque las ideas aún están en mudanza. Las planteo por tanto, como una provocación, un guiño. Aun así, ya percibo que en esta maraña hay ciertas intensidades, ciertos nudos que me gustaría proponer a modo de (in)conclusión.

Entiendo el nudo según lo planteado por la socióloga feminista Julieta Kirkwood (1984), quien propuso la conceptualización de nudos de pensamiento. Para Kirkwood los nudos son sitios de posibilidades, siempre en movimiento que, así como los nudos de los árboles, fuerzan a una nueva geometría. Los nudos son, por lo tanto, más que un obstáculo, una oportunidad de modificar la trayectoria del pensar. Así también, Kirkwood nos propone aproximarnos a los nudos sin prisa, observando el origen de las hebras, soltándolas en el sentido inverso, trabajando con todo el cuerpo uñas y dientes. A mí la conceptualización de nudos, también me despierta a la materialidad de nuestros tejidos humanos, el cuerpo en su entramado de fibras, sus músculos, huesos, fascias, sus ligamentos y tejido nervioso. A los nudos vivos, tejidos y sedimentados en el cuerpo.

Es por ello que para finalizar propondré el nudo: Qué excluye la silla, el cual se acompaña de cuestionamientos, tales como: ¿cuáles son los cuerpos, los movimientos que son invisibilizados por la sillas?, ¿cuál es la potencialidad del aprendizaje que la 
silla excluye?, ¿cuáles son esas posibilidades corporales?, ¿cuáles son las realidades probables de enactuar?

No tengo respuestas. Más bien, una invitación a seguir enmarañándonos, y dejar que la materialidad de nuestras inquietudes abra espacios para fecundas intra-acciones, que a su vez, materialicen acciones que involucren relaciones, diálogo y encuentro. Resulta indispensable movilizarse de la estasis crítica, y es por ello que las palabras de la feminista Julieta Kirkwood (1986: 18) están vigentes aún hoy "necesitamos la confrontación y el juego de las ideas abiertas de par en par, millones de claridades, de pequeñas ideas."

\section{Referencias bibliográficas}

Alaimo, S., \& Hekman, S. (2008). Material Feminism. United States of America: Indiana University Press.

Anzaldúa, G. (1999). Borderlands = La frontera (2nd ed. ed.). San Francisco: San Francisco : Aunt Lute Books.

Barad, K. (2003). Posthumanist Performativity: Toward an Understanding of How Matter Comes to Matter. Signs, 28(3), 801-831.

Barad, K. (2007). Meeting the Universe Halfway Quantum Physics and the entanglement of matter and meaning United States of America: Duke University Press.

Barad, K. (2014). Diffracting Diffraction: Cutting Together-Apart. Parallax, 20(3), 168187. doi: 10.1080/13534645.2014.927623

Blum, L. M., \& Fenton, E. R. (2016). Mothering with Neuroscience in a Neoliberal Age: Child Disorders and Embodied Brains In L. Käll (Ed.), Bodies, boundaries and vulnerabilities : Interrogating social, cultural and political aspects of embodiment: : Springer.

Braidotti, R. (2011). Nomadic Subjects. United States of America: Columbia University Press.

Calvo, C. (2005). Entre la educacion corporal caotica y la escolarizacion corporal ordenada. revista Iberoamericana de Educacion, 39. 
Calvo, C. (2014). ¿Qué pasaría si a los niños y niñas se les dejara aprender? Polis Revista Latinoamericana (online), 27, 2-16. doi: 10.4000/polis.9687

Calvo, C. (2015). Entrevista Sarita Galvez. La Serena, Chile.

Coole, D., \& Frost, S. (2010). Introducing the New Materialisms. In D. Coole \& S. Frost (Eds.), New Materialisms: Ontology, Agency and Politics. United States: Duke University Press.

Cranz, G. (1998). The chair: rethinking culture, bosy and design. United States of America: W.W. Norton \& Company.

Dahlberg, G., \& Moss, P. (2005). Ethics and Politics in Early Childhood Education (Vol. 1). UK: RoutledgeFalmer.

Duhn, I. (2014). Making agency matter: rethinking infant and toddler agency in educational discourse. Discourse: Studies in the Cultural Politics of Education, 1-12. doi: 10.1080/01596306.2014.918535

Grau, O. (2011). Representaciones sociales de la infancia, discursos y prácticas. In F. Cousiño \& A. M. Foxley (Eds.), Políticas Públicas para la Infancia (pp. 43-55). Santiago de Chile: Graficca Lom Ltda.

Haraway, D. (1988). Situated Knowledges: The Science Question in Feminism and the Privilege of Partial Perspective. Feminist Studies, 14(3), 575-599. doi: $10.2307 / 3178066$

Integra, F. (2014). "En el jardín aprendí". 2015, from https://www.youtube.com/watch?v=frOtLjPB-L4

Integra, F. (2015). Fundación Integra Website. 2015, from www.integra.cl

Jackson, A., Mazzei, Lisa A, \& Ebooks Corporation. (2012). Thinking with theory in qualitative research : Viewing data across multiple perspectives (1st ed.] ed.). Abingdon, Oxon ; New York, NY: Routledge.

Kehily, M. J. (2013). Understanding childhood: a cross disciplinary approach (2nd. ed ed. Vol. Childhood). Briston, Milton Keynes: Open University Press, Policy Press. 
Kirkwood, J. (1984). Los nudos del pensamiento feminista. Facultad Latinamericana de Ciencias Sociales, 64.

Kirkwood, J. (1986). Ser politica en Chile: las feministas y los partidos. Santiago de Chile: FLACSO.

Latour, B. (2004). How to Talk About the Body? The Normative Dimension of Science Studies. Body and Society, 10(2-3), 205-229. doi: 10.1177/1357034X04042943

Lenz Taguchi, H. (2010). Going Beyond the Theory/Practice Divide in Early Childhood Education: Introducing an Intra-active pedagogy. United States: Routledge

Letelier, O. (1976). The Chicago Boys in Chile: Economic Freedom's Awful Toll. The Nation.

Maclure, M. (2013). Researching without representation? Language and materiality in post-qualitative methodology. International Journal of Qualitative Studies in Education, 26(6), 658-667. doi: 10.1080/09518398.2013.788755

Muñoz Goma, O. (2007). El Modelo Económico de la Concertación 1990-2005. ¿Reformas o Cambio? . Santiago de Chile: CIEPLAN/FLACSO.

OECD. (2004). Reviews of National Policies for Education: Chile 2004 Reviews of National Policies for Education. Paris, France: OECD.

Olsson, L., \& Ebooks Corporation. (2009). Movement and Experimentation in Young Children's Learning : Deleuze and Guattari in Early Childhood Education. (Contesting Early Childhood). Hoboken: Taylor \& Francis.

Reichle, F. (Writer). (2007). Montegrande featuring Francisco Varela and H.H. Tenzin Gyatso, XIV Dalai Lama. First Run/Icarus Films, New York, 2004. VHS, 80 mins., col.

Taylor, A. (2013). Reconfiguring the natures of childhood. Abingdon, Oxon: Routledge.

Tesar, M. (2016). Policy and philosophy in the contemporary educational landscape. Policy Futures in Education, 14(3), 311-313. doi: 10.1177/1478210316640621

Umayahara, M. (2006). Paper commissioned for the EFA Global Monitoring Report 2007, Strong foundations: early childhood care and education. Education for All 
Global Monitoring Report 2007: United Nations Educational, Scientific and Cultural Organization.

Valdes, T. (1987). Las mujeres y la dictadura militar en Chile. Material de discusion programa FLACSO-Santiago de Chile, 94.

Valdivia Ortiz de Zarate, V. (2010). ¡ESTAMOS EN GUERRA, SEÑORES!: EL RÉGIMEN MILITAR DE PINOCHET Y EL "PUEBLO", 1973-1980. . Historia, 43(1), 163-201.

Varela, F. (2000). Steps to a Science of Inter-being: Unfolding the Dharma Implicit in Modern Cognitive Science. In G. Watson, S. Batchelor \& G. Claxton (Eds.), The Psychology of Awaking: Buddhism, Science, and Our Day-to-Day Lives. Canada: Red Wheel/Weiser

Varela, F., \& Poerksen, B. (2006). Truth Is What Works : Francisco J. Varela on Cognitive Science, Buddhism, the Inseparability of Subject and Object, and the Exaggerations of Constructivism--A Conversation. The Journal of Aesthetic Education, 40(1), 35-53.

Varela, F. J. (1999). Ethical know-how: Action, wisdom, and cognition: Stanford University Press.

Varela, F. J., Rosch, E., \& Thompson, E. (1991). The embodied mind: Cognitive science and human experience: MIT press. 